\title{
Cardiometabolic Health Risks, Lifestyle Behaviours and Quality of Life in People with Early Psychosis - A cross sectional study
}

Assist. Prof. Dr. Soontareeporn Meepring ${ }^{1}$, Prof. Richard Gray ${ }^{2}$, Dr. Yan Li ${ }^{3}$ Dr Grace W.K. Ho ${ }^{3}$, Prof. WaiTong Chien ${ }^{4},{ }^{*}$ Prof. Daniel Bressington ${ }^{5}$

${ }^{1}$ Naresuan University, Phitsanulok, Thailand; ${ }^{2}$ La Trobe University, Melbourne, Australia; ${ }^{3}$ The Hong Kong Polytechnic University, Hong Kong SAR, PRC; ${ }^{4}$ The Chinese University of Hong Kong, Hong Kong SAR, PRC; ${ }^{5}$ Charles Darwin University, Darwin, Australia.

* corresponding author: Prof Daniel Bressington, College of Nursing and Midwifery, Charles Darwin University, Casuarina, Darwin, Australia. Email: daniel.bressington@cdu.edu.au

Trial registration: 3rd November 2017 (www.thaiclinicaltrials.org; study ID: TCTR20180305002).

Funding: This study was supported by a research grant from The Thailand Research Fund and the Office of The Higher Education Commission, Royal Thai Government, contract No. MRG6080216.

Key words: Early Psychosis; Cardiovascular Disease Risk; Quality of Life; Lifestyle Behaviours.

\section{Abstract:}

Introduction: People with schizophrenia have poor cardiometabolic health, with elevated 10-year cardiovascular disease risk (CVD-R) scores and poor quality of life (QOL). There is lack of understanding of these issues in early psychosis.

Aims: To quantify CVD-R in people with early psychosis and profile their obesity prevalence, lifestyle behaviours and QOL. Secondary aim was to explore associations between lifestyle behaviours/treatment characteristics and CVD-R/QOL.

Method: Baseline data from 81 RCT participants were used to profile cardiometabolic health risks (QRISK ${ }^{\circledR}$, BMI and waist circumference). Participants self-reported lifestyle behaviours and QOL. Relationships between modifiable treatment/lifestyle factors and QOL/CVD-R were explored using bivariate analyses.

Results: Participants' relative risk for CVD over 10 years was 1.93 times higher than healthy counterparts; $39 \%$ also had an obese BMI and physical QOL was poor. No significant associations were observed between CVD-R or QOL with treatment characteristics and lifestyle factors.

Discussion: Despite positive lifestyle behaviours, participants had elevated CVD-R scores and poor physical health related QOL. Quantifying CVD-R with $\mathrm{QRISK}^{\circledR} 3$ may highlight the need for health promotion interventions.

Implications for practice: Mental health professionals should be alert to elevated cardiometabolic health risks in early psychosis, particularly as these can occur in the context of relatively healthy lifestyle behaviours. 


\section{Accessible summary}

\section{What is known on the subject?}

- $\quad$ People with schizophrenia have poor cardiometabolic health, with elevated 10-year cardiovascular disease risk (CVD-R) scores and low quality of life (QOL).

- $\quad$ There is a lack of understanding about CVD-R scores in people with early psychosis and no studies have quantified CVD-R using the $\mathrm{QRISK}^{\circledR} 3$ calculator in this client group.

- $\quad$ Establishing potential relationships between modifiable lifestyle behaviours/treatment characteristics with CVD-R or QOL may identify targets for early intervention.

\section{What the paper adds to existing knowledge?}

- $\quad$ This is the first study to quantify the individual 10 -year CVD-R of people with early psychosis utilising the $\mathrm{QRISK}^{\circledR 3}$ calculator.

- We observed low levels of physical health related QOL and high levels of CVR-R despite participants reporting relatively positive lifestyle behaviours.

\section{What are the implications for practice?}

- $\quad$ The cardiometabolic health state of this client group warrants as much attention as for those with an enduring severe mental illness; early preventative interventions are warranted.

- $\quad$ It may be useful to routinely quantify the CVD-RR of people with early psychosis using the QRISK ${ }^{\circledR 3}$ calculator, even in the absence of immediate concerns about lifestyle behaviours. 


\section{Cardiometabolic Health Risks, Lifestyle Behaviours and Quality of Life in People with Early Psychosis - A cross sectional study}

\section{Introduction}

People diagnosed with severe mental illness (SMI) are disproportionally affected by a range of cardiometabolic disorders (Olfson et al., 2015; Vancampfort et al., 2014; Correll et al., 2017). Around onehalf of people with SMI are likely to be obese, approximately a fifth will present with type 2 diabetes and more than one-half will meet the criteria for metabolic syndrome (Gardner-Sood et al., 2015; Olfson et al., 2015; Mitchel et al., 2013). Metabolic syndrome and obesity directly contribute to considerably increased rates of early mortality (Nordentoft et al., 2013; Tihonen et al., 2009), often from cardiovascular disease.

\subsection{Cardiovascular disease risk in severe mental illness}

A meta-analysis of observational studies including over 3 million participants (Correll et al., 2017) concluded that people with SMI have significantly higher odds of coronary heart disease (OR=1.51, 95\% $\mathrm{Cl}: 1.47-1.55)$ and cardiovascular disease (CVD) (OR=1.53, 95\% Cl: 1.27-1.83) compared with the general population. Some studies that calculate CVD risk scores using algorithms have also reported that people diagnosed with SMI have increased CVD risk when compared to healthy controls. For example, Kilicaslan et al. (2019) concluded that the 10-year cardiovascular risk of 103 Turkish inpatients with schizophrenia was significantly higher than 39 healthy matched controls $(5.16 \%$ vs. 3.02\%, $p=0.03)$. Similarly, a Chinese study involving 466 inpatients with schizophrenia (Zhao et al., 2018) reported that their Framingham 10year CVD risk score was significantly higher than the 507 healthy controls $(6.7 \%$ vs. $4.8 \%, p<0.001)$. Another study of 82 Hong Kong Chinese adults with SMI (who had a mean duration of illness of around 20 years) reported that $13 \%$ of participants had a moderate-to-high 10 -year CVD QRISK ${ }^{\circledR 2}$ risk score (Bressington et al.,2016).

\subsection{Cardiovascular disease risk scores in early psychosis}

Very few studies have calculated cardiovascular risk scores in people with early psychosis (i.e. within 5 years of onset; Breitborde et al., 2009). This is an important gap in evidence as establishing 10-year CVD risk would help to inform the development of preventative interventions, health services, resources planning and policy (Hippisley-Cox et al., 2017). One relevant study (Phutane et al., 2011) concluded that while 56 participants with early psychosis in the United States had significantly higher rates of smoking and hypertension than 146 gender- and age-matched healthy controls, their 10-year risk of developing CVD was not significantly different. However, further studies are required to corroborate this finding, particularly in other countries, as the development and prevalence of cardiometabolic health risks has been reported to vary in accordance with study setting and culture (Tek at al., 2016). Studies using the most preferred and up-to-date CVD risk calculators in people with early psychosis are also required. The British Association for Psychopharmacology (Cooper et al., 2016) and the UK's National Institute for Health and Clinical Care Excellence NICE guideline CG181 suggest using the QRISK ${ }^{\circledR} 2$ calculator to assess CVD in 
people with SMI (NICE, 2014), but a more recent surveillance report advised to replace this with QRISK $^{\circledR 3}$ due to its greater potential value in identifying at-risk patients (NICE, 2018). To the best of our knowledge, no studies have been conducting using the QRISK ${ }^{\circledR 3}$ (Hippisley-Cox et al., 2017) in patients with early psychosis.

\subsection{Lifestyle and treatment-related factors associated with CVD}

It is beneficial to ascertain factors associated with elevated CVD risk scores in people with early-stage psychosis to identify potential targets for early intervention and predictors of underlying CVD risk. Studies reported that many modifiable factors, including those related to prescribed medications, could be associated with high rates of cardiometabolic disorders in people with longer-term SMI. For example, there is evidence of a positive association between antipsychotic polypharmacy, duration of exposure to antipsychotics and cardiovascular disease mortality (Ganguly et al., 2004; Weinmann et al., 2009). Likewise, a few studies have also demonstrated that elevated CVD risk score is associated with taking higher doses of antipsychotics (Bressington et al., 2016) and usage of multiple types of antipsychotics (Killiscan et al., 2019). However, these studies did not involve people with early-stage psychosis.

The risk of developing cardiometabolic disorders among people with SMI is also compounded by their increased likelihood to adopt unhealthy lifestyle behaviours, such as poor diet, tobacco smoking, excessive alcohol consumption, and lack of exercise (Vermeulen-Smit et al., 2015). Due to a lack of studies, the associations between lifestyle behaviours and CVD risk indicators in early psychosis remains uncertain. Although a recent prospective study conducted in the UK with 293 adults with early psychosis (Gaughran et al., 2019) found no relationships between lifestyle factors and types of antipsychotics with cardiometabolic health state at either baseline or at one-year follow up, further studies are needed to replicate these findings in other psychosis populations and care settings.

\subsection{Lifestyle and treatment-related factors associated with quality of Life}

Being able to identify which modifiable treatment and lifestyle factors are associated with subjective quality of life (QOL) would help to inform interventions for people with early psychosis, however limited evidence is available. This is an important gap in understanding as QOL measures are increasingly considered as being vital in both the planning and evaluation of interventions designed to improve the health outcomes of people with SMI (Chien at al., 2020), particularly in early psychosis (Theodore et al., 2010). To date, most studies in early psychosis have measured relationships between QOL and illnessrelated characteristics (Renwick et al., 2017), but the associations of QOL with health-related behaviours are still poorly understood. A few studies conducted in people with longer-term SMI have provided some indications that these modifiable lifestyle factors may be associated with QOL. For example, Costa et al (2018) reported that sedentary time was associated with lower environmental and social QOL, whereas greater physical activity levels predicted higher physical and global QOL when measured using the WHOQOL-BREF in a cross-sectional survey conducted with 115 people with schizophrenia.

\subsection{Study objectives}


The primary objective of this study was to quantify the individual 10-year CVD-RR in a sample of Thai people with early psychosis utilising the $\mathrm{QRISK}^{\circledR 3}$ calculator, and profile their obesity prevalence, healthy lifestyle behaviours, and QOL. A secondary objective was to provide a preliminary examination of the associations between modifiable lifestyle and treatment characteristics with CVD-RR and QOL. Establishing the relationships between modifiable factors and CVD-RR/QOL are expected to help identify potential targets for interventions.

\section{Materials and methods:}

\subsection{Study design}

This is a nested cross-sectional study using baseline data from the participants of an ongoing RCT entitled "Thai Health Improvement Profile intervention to prevent obesity in people with early psychosis". The RCT was prospectively registered with the Thai Clinical Trials Registry (www.thaiclinicaltrials.org) on 3rd November 2017 (study ID: TCTR20180305002). The current study is reported in accordance with STROBE reporting guidelines for observational studies (Von Elm et al. 2007).

\subsection{Study setting}

The study was conducted in an outpatient department of a large public psychiatric hospital in the Northern part of Thailand, providing general psychiatric services for a local population of around three million people. The outpatient service was staffed by a multidisciplinary team, including registered nurses, psychiatrists and psychologists, who saw approximately 200 service users per day. The recruitment for the RCT and baseline data collection were conducted from 10/2018 to 04/2020.

\subsection{Subjects}

Inclusion criteria of participants were those who were:

a) recently (no more than 5 years), diagnosed with any psychotic illness by a psychiatrist; b) receiving any antipsychotic medication - either alone or in combination with other psychotropic medications; c) Thai adult residents ( $\geq 18$ years) receiving treatment/care at the hospital out-patient clinic; c) able to understand Thai and provide written informed consent, and: d) considered safe and competent to participate in the study, as suggested by the responsible psychiatrist.

Potential participants were excluded if they:

a) had co-morbidity of a chronic physical illness or disability (such as major learning disability, organic brain diseases or Type 1 diabetes); b) had a primary diagnosis of substance misuse disorder; c) planned or expected to terminate the antipsychotic medication at recruitment; $d$ ) were receiving any other physical health monitoring or intervention programme beyond usual care (except if the intervention was primarily for their mental health, such as ECT).

\subsubsection{Sample size}


We did not calculate an a-priori sample size as we planned to analyse all available data collected at baseline in the ongoing RCT.

\subsection{Ethical considerations and approval}

The study was granted ethical approval by the Nakhon Sawan Rajanagarindra Psychiatric Hospital (reference number 07/2560) prior to commencement of the project. The study was explained in detail to the potential participants and their family members (if present) and they were also given written information. They were asked to provide their written informed consent after being given time to consider their decision. They were aware that their participation was voluntary and that they could withdraw from the study at any time. They were also informed that declining the invitation to take part would have no detrimental effect on their routine treatment or damage their relationships with clinical staff.

\subsection{Recruitment and data collection}

Potential participants were identified by screening their medical records. Those meeting the study inclusion criteria and considered safe to participate by their psychiatrists were approached by the research nurse when they attended their next scheduled outpatient appointment (i.e. using convenience sampling). Trained community nurses working in the outpatient setting gathered the data after the participants provided their written informed consent and populated a data collection form designed specifically for this study.

\subsection{Study variables}

\subsubsection{Demographic and clinical characteristics}

Data regarding demographic and clinical characteristics were retrieved from the participants' clinical records (where available) and checked with the participants and their carer as necessary. These data consisted of sex, age, employment status, education level, marriage status, psychiatric diagnosis, duration of illness, prescribed psychiatric treatments, prescribed physical health medications (i.e. for hypertension, type 2 diabetes, dyslipidaemia) and cholesterol/fasting blood glucose levels if recorded within the prior month.

\subsubsection{Objective measures of physical health state}

The nurses recorded participants' height, weight, pulse, waist circumference and blood pressure using standardised procedures and regularly calibrated equipment that is used routinely in the outpatient's clinic. We used the cut-off point categories for Asians to indicate risk, including underweight (BMI $<18.5)$, normal $(18.5 \geq \mathrm{BMI}<23)$, overweight at risk $(23 \geq \mathrm{BMI}<25)$, obese I $(25 \geq \mathrm{BMI}<30)$ and obese II (BMI $\geq 30$ ), in accordance with the International Obesity Task Force (Kanazawa et al. 2002) and World Health Organization Expert Consultation (2004). We also used the International Diabetes Federation (IDF, 2006) ethnicity specific central obesity waist circumference measurement cut-off values of $\geq 90 \mathrm{~cm}$ (men) and $\geq 80 \mathrm{~cm}$ (women).

\subsubsection{Cardiovascular disease risk}


We calculated the 10-year individual cardiovascular risk of participants aged over 24 years with the QRISK ${ }^{\circledR 3}$-2018 Web Calculator (at: https://qrisk.org/three/) using the physiological data (i.e. BMI, data routinely recorded in the medical notes). The QRISK ${ }^{\circledR 3}$ tool was further developed from the previous QRISK $^{\otimes 2}$ by including additional risk variables in order to provide valid measures of absolute risk (Hippisley-Cox et al., 2017). The QRISK ${ }^{\circledR 3}$ algorithm calculated a person's risk of developing stroke/heart attack over the next 10 years (as a percentage), and the relative risk (CVD-RR) for the individual by dividing their percentage risk by the score of a healthy person with the same age, sex, and ethnicity (Hippisley-Cox et al., 2017). Other examples of information that were entered into the calculator included smoking status/level, taking atypical antipsychotic medication, diagnosis of severe mental illness, height, weight, diabetes status, cholesterol/HDL ratio, and other relevant medical histories. The calculator imputed estimated data where fields were left blank due to missing information. For data analyses, we dichotomized participants into two groups ( $0=$ no elevated risk or $R R \leq 1$; and $1=$ with elevated risk or $R R>1)$.

\subsubsection{Lifestyle behaviours}

Participants (and their family carer) self-reported their healthy lifestyle behaviours (including dietary habits [daily vegetable/fat intake], weekly exercise levels, alcohol use and smoking status). Each healthy lifestyle behaviour was rated as green (healthy) or red (unhealthy) in accordance with the HIP-T red flagged cut-off points described by Meepring at al., (2018): ( $\leq 2$ portions fruit/vegetables a day; $\geq 2$ portions fat a day; $<30$ min of activity 5 days a week; $\geq 2$ alcohol units/day (Women) and $\geq 3$ alcohol units/day (Men); smokes tobacco). For data analyses we coded each of the five areas of lifestyle behaviour variables as $0=$ healthy (green) and $1=$ unhealthy (red).

\subsubsection{Quality of life}

Patients' self-reported health related quality of life was measured with the Thai language version of WHOQOL-BREF (Whoqol Group, 1998). This version of the tool is the official measure of quality of life outcomes used by Thai Department of Mental Health, Ministry of Public Health (Lim and Zebrac, 2004). It was reported to have good psychometric properties, and a valid and reliable measure of health-related quality of life in a variety of Thai sub-populations; for example, internal consistency for the four domains ranged from $\alpha=0.73-0.83$ in Thai college students (Li et al., 2009). The measure contained 26 questions that were rated on a 5 -point Likert scale (i.e. from " 1 very poor", to " 5 very good"; or " 1 not at all", to " 5 an extreme amount"). It was scored on 4 domains (physical health, psychological health, social relationships and environment) and two separate facets (Overall quality of Life and General Health), with a higher score indicating a better quality of life. We converted the raw domain scores of WHOQOL-BREF to WHO 0-100 scores as recommended by WHO (1996).

\subsection{Statistical analysis.}

All data were analysed using IBM SPSS for Windows, version 23.0. The level of statistical significance was set at $p<0.05$ for all tests (two-tailed). Descriptive statistics were used to summarize the sociodemographic information, clinical characteristics, healthy lifestyle behaviours, physiological data, CVD 10year risk score (percentage), CVD-RR and WHOQOL-BREF domain scores. We recoded the modifiable 
treatment variables into $0=y e s$ and $1=$ no (i.e. for being prescribed: clozapine; long acting antipsychotic; second generation antipsychotic; polypharmacy).

To address our secondary objective, we conducted exploratory bivariate analyses to identify significant associations between the modifiable treatment/lifestyle behaviour variables with CV-RR and the four WHOQOL-BREF domains. With considerations of small sample size, and potentially small cell counts and skewness in data, non-parametric tests (i.e. Chi-square or Fisher's exact) were used to examine bivariate associations between increased CVD-RR (yes/no) with the treatment variables (clozapine; long acting antipsychotic; second generation antipsychotic; polypharmacy) and lifestyle variables (fruit/vegetables intake; fat intake; exercise; alcohol intake; smoking). Whereas, we used Mann-Whitney U-tests to examine the distribution of the for WHO-QOL domain scores across the treatment and lifestyle categories. Given the multiple comparisons conducted, we adjusted the significance level to reduce the risk of a type I error (i.e. by dividing 0.05 by the number of comparisons).

\section{Results}

A total of 145 service users with a relatively recent diagnosis of a psychotic disorder were screened to establish if they met the inclusion criteria for the ongoing RCT. Of these, 33 were excluded as they were: diagnosed with psychosis for $>5$ years $(n=23)$; too unwell to provide informed consent $(n=5)$; and/or involved in another physical intervention study beyond usual care $(n=5)$. Six of the remaining 112 potential participants declined to take part (i.e., overall response rate for eligible participants was 93\%). Finally, 106 proceeded to be randomised for the RCT. A total of 25 participants were excluded from the present analyses as they were aged $<25$ years and hence too young for the QRISK ${ }^{\circledR} 3$ calculator, resulting in a final sample of 81 participants.

\subsection{Demographic and Clinical Characteristics}

Full details of the demographic and clinical characteristics are presented in table 1.

Half of the participants were male, with an average age of 40 years and a mean duration of illness of just over 2 years (range 1-5 years). Just under half were single, a third were married and the remainder were either divorced or widowed. There was a relatively low level of academic achievement, with around half of the participants completed a primary school level of education or below and only one in ten finished a programme of study at college or university. More than half were not in paid employment.

Almost a half of participants (47\%) had a diagnosis of "other non-organic psychotic disorders", a third had a diagnosis of schizophrenia and the rest were diagnosed with either "acute and transient psychotic disorder", "schizoaffective disorders" or "unspecified non-organic psychosis". All participants were prescribed a least one antipsychotic medication, with a mean of 1.26 types ( $s d=0.47$, median=1, range 13). Two-thirds were taking second-generation anti-psychotics and just under a quarter prescribed antipsychotic polypharmacy. Clozapine was prescribed for around $10 \%$ of participants; and just under one 
quarter were receiving long acting antipsychotic injections. Very few participants were prescribed medications for physical health conditions.

Please insert table 1 here.

\subsection{Cardiometabolic health risks (CVD RR, obesity prevalence)}

Over half of the participants $\left(59 \%, n=48\right.$ ) were found to have an at-risk BMI ( $\geq 23 \mathrm{kgm}^{2}$ ); and $39 \%$ ( $n=32$ ) had a BMI within the obese I or II range. Further, $44 \%$ of males and $60 \%$ of females had a waist circumference measurement indicating central obesity.

The individual CVD 10-year percentage risk score ranged from $0.10-14.90 \%$ and only one participant was found to have a score suggesting a moderate-high CVD risk (i.e. $\geq 10 \%$ ). However, the mean CVD-RR (risk of developing stroke/heart attack over the next 10 years relative to a healthy person with the same age, sex and ethnicity) was 1.93 ( $s d=1.55$, range $0.70-12.30)$; and the majority $(80 \%, n=65$ ) were found to have an elevated CVD-RR (RR of $>1.0)$.

Please see table 2 for details of cardiometabolic health risks, self-reported healthy lifestyle behaviours and subjective QOL.

\subsection{Health behaviours}

In terms of self-reported lifestyle behaviours, only one-third $(n=27)$ of the participants lacked exercise, $28 \%$ smoked tobacco, and about one-tenth ate too much fat (12\%) and drank excessive amounts of alcohol (10\%). Very few (7\%) ate insufficient fruits/vegetables.

\subsection{Subjective QOL}

The average transformed WHOQOL-BREF 100 score was highest in the environment domain (61.59, $s d=11.69$ ) and lowest in the social relationship domain $(49.67, s d=16.26)$. Whereas, the physical health and psychological health domain mean scores were very similar $(54.74, \mathrm{sd}=11.35$ and 56.90 ; $\mathrm{sd}=11.82$, respectively).

\subsection{Relationships between modifiable lifestyle and treatment variables with CVD-RR and QOL}

No statistically significant associations were identified between CVR-RR or WHOQOL-BREF domain scores with modifiable lifestyle and treatment variables (all $p$ values $>0.05$ ).

\section{Discussion}

The primary objective of this study was to profile the cardiometabolic health risks, self-reported healthrelated behaviours and subjective QOL in a community-based sample of Thai people with early-stage psychosis. Overall, more than half (59\%) of the participants were found to have an at-risk BMI ( $\left.\geq 23 \mathrm{kgm}^{2}\right)$; and central obesity was also common ( $44 \%$ of males and $60 \%$ of females). This was despite the fact that 
they reported generally positive healthy lifestyle behaviours (with the exception of a third that did not exercise sufficiently). This finding concurs with that of a previous study (Thongsai et al., 2016) conducted in southern semi-rural Thailand involving 105 people diagnosed with schizophrenia (with a longer average duration of psychosis of 4 years). The study reported that although healthy lifestyle behaviours were comparatively better than those reported in the UK and the USA, at-risk BMI values were still observed in 44\% of participants (Thongsai et al., 2016; Bos et al.,2021). However, the current cohort seemed to have somewhat higher proportions of participants with an at-risk BMI value.

The comparatively high proportion of participants in the current study with an at-risk BMI is noteworthy because they had relatively good health behaviours and a short duration of diagnosis/treatment (around 2 years). However, research evidence demonstrated there is a high potential for rapid weight gain in people recently diagnosed with a psychosis. A systematic review of 25 studies from different countries demonstrated that cardiometabolic risk factors (including weight gain) could increase within a month after the first exposure to antipsychotic drugs (Foley and Morley, 2011). Weight-gain has also been found to be much faster and higher in younger people and those not previously exposed to antipsychotic drugs (Homel et al., 2002). An increase of up to $4.5 \mathrm{~kg}$ of body weight has been reported after taking 10-12 weeks of second-generation antipsychotics (Patel et al., 2009). Therefore, the current study corresponded with these earlier findings; and indeed, it has highlighted that it is essential to closely monitor and manage weight gain, particularly in those who have recently started taking antipsychotic drugs

The results of the current study revealed that participants had an elevated CVD-RR (mean=1.94; median 1.4) to develop a cardiovascular event over the next 10 years relative to a matched healthy person. Due to a lack of similar studies conducted using QRISK calculators in people with early psychosis, it is difficult to make any direct comparison. However, this level of relative risk is certainly lower than the mean CVDRR of 2.80 ( $\mathrm{sd}=2.96$ ) and median of 1.80 (IQR=1.87) using QRISK2 reported in an observational study of 82 people with chronic SMI (average duration of 8.5 years illness) in Hong Kong (Bressington et al., 2016). It is possible that the lower CVR-RR in the current study could be attributed to the shorter duration of illness, hence having less exposure to antipsychotics. It is also possible that the relatively positive lifestyle behaviours reported by participants in the current study (particularly the healthy diet of most and active lifestyle of two thirds) have gone some way towards improving their cardiometabolic health state.

The participants in the current study have reported a reasonably good environment related QOL (mean of 61.6), but a low level of social QOL. However, the population norms of the WHOQOL-BREF are currently not available for Thailand so it is unclear how the participants' QOL in this study compares to the QOL of similar-aged Thai people without early-stage psychosis. As might be expected, the QOL domain scores in the current study were far less than those of 470 Thai college students without mental disorders (mean age 20.5 years), reporting the WHOQOL-BREF domain mean scores between 63 and 69 (Li et al., 2009).

In terms of studies involving people with an enduring mental illness, a study conducted in Thailand (Suttajit and Pilakanta, 2015) with 80 people diagnosed with schizophrenia reported better physical (63 vs 54.7) and psychological (66 vs 56.9) WHOQOL-BREF scores than the current study, but lower scores for the other domains. Notably, while the patients with schizophrenia in Suttajit and Pilakanta's (2015) study had 13.6 years of average duration of illness and similar mean age, the current cohort had considerably 
lower mean scores of QOL. However, this may be partially explained by the very low mean total PANSS score of 45.6 among the participants in Suttajit and Pilakanta's study, suggesting low levels of psychotic symptoms. Perhaps the most directly comparable study to ours used the WHOQOL-BREF with 146 Irish outpatients with first-episode non-affective psychosis (Renwick et al., 2012). When compared to the current study, Renwick et al., described better scores for physical (58.5), comparable scores for environment domain (59.5), but considerably lower scores for psychological (45.5) and social (43.9) QOL. The differences in physical health related QOL observed between the two studies could be attributed by the younger age (mean=33 years) of the participants in Renwick et al.'s (2012) study. It could also be likely that the participants in Renwick et al.'s study had less physical health concerns because they had only started receiving treatment; whereas, the participants in the current study had been prescribed antipsychotic medications for an average of two years, which could have potentially more detrimental effects on physical health as time went by.

The second objective of this study was to explore relationships between CVD RR/QOL with healthy lifestyle behaviours and characteristics of antipsychotic treatment. We found no significant associations between treatment characteristics or health behaviours and CVD-RR, similar to Gaughran et al.'s (2019) UK study of people with early psychosis showing no relationship between lifestyle factors, types of antipsychotics and cardiometabolic health state. However, the lack of relationships observed in the current study between QOL and lifestyle behaviours is at odds with several studies involving people with longer term severe mental illnesses showing that physical activity is positively associated with some aspects of QOL (Costa et al., 2018; Deenick et al., 2017; Vancampfort et al., 2011).

The absence of significant associations observed between lifestyle factors, treatment characteristics and cardiovascular health/QOL in the current cohort needs further research with a larger representative sample of people with early psychosis. The current cohort is relatively homogenous in terms of their lifestyle behaviours and BMI, which may account for the failure to identify significant relationships between these variables. Future research should also be conducted with different ethnic groups because the development of CVD risks varies in accordance with ethnicity (Hippisley-Cox et al., 2017); and the extent of cardiometabolic health risks following treatment with antipsychotics was found to be lower in Asians with early psychosis than the western counterparts (Tek at al., 2016).

\subsection{Implications for mental health nursing practice and research}

The results of this study demonstrate that it is possible for people with early psychosis and a short duration of treatment to have an increased risk of CVD and low levels of physical health related QOL in the context of relatively healthy lifestyle behaviours. Mental health nurses and other professionals need to appreciate that poor cardiometabolic health can develop soon after psychosis onset even when relatively positive lifestyle behaviours are adopted. These findings indicate that the cardiometabolic health state of this client group warrants as much attention as that provided for people with an enduring severe mental illness and that early preventative interventions may be warranted. In this regard, it may be useful to routinely quantify the CVD-RR of people with early psychosis using the QRISK ${ }^{\circledR 3}$ calculator, even when concerns about unhealthy lifestyle behaviours have not been reported or observed. 
Future studies should explore if sharing the CVD-RR score results with service users and their families may be of benefit in a lifestyle counselling programme to raise awareness of the need to change and to evaluate intervention outcomes. Although we did not observe relationships between objective/subjective physical health state with modifiable treatment and lifestyle factors in the current study, this may be because the potentially detrimental impact of treatment and unhealthy behaviours had yet to manifest due to the short duration of illness. Subsequent observational studies with a larger representative sample could also further investigate the apparent lack of relationships between lifestyle factors and cardiovascular health/QOL observed in the current cohort, perhaps by adopting a longitudinal design to analyse changes over time.

\subsection{Study limitations}

This study had a few main limitations requiring attention when interpreting the results. This was a convenience sample of Thai people with early psychosis at one outpatient clinic; and thus, the sample was not representative of this patient group in Thailand and the generalisability of the results is limited. Second, the lifestyle behaviours were self-reported by participants (often in the presence of their family carers) and thus subject to social desirability reporting bias. In addition, due to financial and resource constraints, limited blood tests were routinely conducted to estimate the cholesterol/HDL ratio in the service or by the research team due to limited funding obtained. Lastly, limited information was available in the medical notes in relation to concurrent physical illness or relevant family medical history. Therefore, the QRISK ${ }^{\circledR}$ 3-2018 Web Calculator had to impute estimated data where the fields were left blank, likely resulting in somewhat underestimation of the participants' CVD risk.

\section{Conclusion}

To the best of our knowledge this was one of very few studies to provide an estimate of 10-year CVD relative risk in a community-based sample of people with early psychosis, and the first to do so using the QRISK3 calculator. The study findings indicated that although the participants self-reported generally positive healthy lifestyle behaviours, they had nearly two times higher risk of developing stroke/heart attack over the next 10 years relative to a healthy person; many of them were also overweight or obese. However, no significant associations between modifiable treatment/lifestyle factors and CDV-RR or QOL were observed in the present sample. Overall, the results indicate that mental health professionals should be alert to the elevated cardiometabolic health risks in people with early psychosis, particularly as these might occur in the context of relatively healthy lifestyle behaviours.

\section{Funding:}

This study was supported by a research grant from The Thailand Research Fund and the Office of The Higher Education Commission, Royal Thai Government, contract No. MRG6080216. 


\section{References:}

Bos, D., Gray, R., Meepring, S., White, J., Foland, K., Bressington, D. (2021). The Health Improvement Profile (HIP) for people with Severe Mental Illness: Feasibility of a secondary analysis to make international comparisons. Journal of Psychiatric and Mental Health Nursing, 2021. Doi: 10.1111/jpm.12748

Bressington, D., Mui, J., Tse, M. L., Gray, R., Cheung, E. F., \& Chien, W. T. (2016). Cardiometabolic health, prescribed antipsychotics and health-related quality of life in people with schizophrenia-spectrum disorders: a cross-sectional study. BMC Psychiatry, 16(1), 411.

Breitborde, N. J., Srihari, V. H., \& Woods, S. W. (2009). Review of the operational definition for firstepisode psychosis. Early intervention in psychiatry, 3(4), 259-265.

Chien, WT, Thompson, DR, Leung SF, Bressington, D. (2020). Quality of life, symptom severity and level of functioning in people with severe mental illness ready for hospital discharge. Journal of Psychiatric and Mental Health Nursing, 2020. Doi: 10.1111/jpm.12703

Cooper, S. J., Reynolds, G. P., Barnes, T. R. E., England, E., Haddad, P. M., ... \& Smith, J. (2016). BAP guidelines on the management of weight gain, metabolic disturbances and cardiovascular risk associated with psychosis and antipsychotic drug treatment. Journal of Psychopharmacology, 30(8), 717-748.

Correll CU, Detraux J, De Lepeleire J, De Hert M. (2015) Effects of antipsychotics, antidepressants and mood stabilizers on risk for physical diseases in people with schizophrenia, depression and bipolar disorder. World Psychiatry. 14:119-36

Correll, C. U., Solmi, M., Veronese, N., Bortolato, B., Rosson, S., Santonastaso, P., ... \& Stubbs, B. (2017). Prevalence, incidence and mortality from cardiovascular disease in patients with pooled and specific severe mental illness: a large-scale meta-analysis of 3,211,768 patients and $113,383,368$ controls. World Psychiatry, 16(2), 163-180.

Costa, R., Bastos, T., Probst, M., Seabra, A., Abreu, S., Vilhena, E., ... \& Corredeira, R. (2018). Association of lifestyle-related factors and psychological factors on quality of life in people with schizophrenia. Psychiatry research, 267, 382-393.

Deenik, J., Kruisdijk, F., Tenback, D., Braakman-Jansen, A., Taal, E., Hopman-Rock, M., ... \& van Harten, P. (2017). Physical activity and quality of life in long-term hospitalized patients with severe mental illness: a cross-sectional study. BMC psychiatry, 17(1), 1-11.

Foley, D. L., \& Morley, K. I. (2011). Systematic review of early cardiometabolic outcomes of the first treated episode of psychosis. Archives of General Psychiatry, 68(6), 609-616.

Ganguly R, Kotzan JA, Miller LS, Kennedy K, Martin B. (2004) Prevalence, trends, and factors associated with antipsychotic polypharmacy among Medicaid-eligible schizophrenia patients, 1998-2000. J Clin Psychiatry. 2004;65(10):1-12. 39. 
Gardner-Sood, P., Lally, J., Smith, S., Atakan, Z., Ismail, K., Greenwood, K. E., ... \& Gaughran, F. (2015). Cardiovascular risk factors and metabolic syndrome in people with established psychotic illnesses: baseline data from the IMPaCT randomized controlled trial. Psychological medicine, 45(12), 2619-2629.

Gaughran, F., Stahl, D., Stringer, D., Hopkins, D., Atakan, Z., Greenwood, K., ... \& Ismail, K. (2019). Effect of lifestyle, medication and ethnicity on cardiometabolic risk in the year following the first episode of psychosis: prospective cohort study. The British Journal of Psychiatry, 215(6), 712-719.

Hippisley-Cox, J., Coupland, C., \& Brindle, P. (2017). Development and validation of QRISK3 risk prediction algorithms to estimate future risk of cardiovascular disease: prospective cohort study. British Medical Journal, 357.

Homel, P., Casey, D., \& Allison, D. B. (2002). Changes in body mass index for individuals with and without schizophrenia, 1987-1996. Schizophrenia research, 55(3), 277-284.

International Diabetes Federation consensus workshop (2006). The IDF consensus worldwide definition of the METABOLIC SYNDROME. International Diabetes Federation.

Kanazawa, M., Yoshiike, N., Osaka, T., Numba, Y., Zimmet, P., \& Inoue, S. (2002). Criteria and classification of obesity in Japan and Asia-Oceania. Asia Pacific Journal of Clinical Nutrition, 11(s8), S732-S737.

Kilicaslan, E. E., Karakilic, M., \& Erol, A. (2019). The Relationship between 10 Years Risk of Cardiovascular Disease and Schizophrenia Symptoms: Preliminary Results. Psychiatry investigation, 16(12), 933.

Li, K., Kay, N. S., \& Nokkaew, N. (2009). The performance of the World Health Organization's WHOQOLBREF in assessing the quality of life of Thai college students. Social indicators research, 90(3), 489-501.

Lim JW, Zebrack B. (2004). Caring for family members with chronic physical illness: a critical review of caregiver literature. Health Qual Life Outcomes. 2004;2:50.

Meepring, S., Chien, WT., Gray, R., Bressington, D. (2018). The effects of the Thai Health Improvement Profile intervention (HIP-T) on the physical health and health behaviours of people with schizophrenia: A quasi experimental study. International Journal of Mental Health Nursing, 27, 126-137.

Mitchell, AJ, Vancampfort, D, Sweers, K, van Winkel, R, Yu, W, De Hert, M (2013). Prevalence of metabolic syndrome and metabolic abnormalities in schizophrenia and related disorders - a systematic review and meta-analysis. Schizophrenia Bulletin 39, 306-318

National Institute for Health and Care Excellence (2014). Lipid modification: cardiovascular risk assessment and the modification of blood lipids for the primary and secondary prevention of cardiovascular disease, NICE guidelines [CG181]. http://www.nice.org.uk/Guidance/CG181

National Institute for Health and Care Excellence (2018). Surveillance report 2018-Cardiovascular disease: risk assessment and reduction, including lipid modification (2014) NICE guideline CG181. http://www.nice.org.uk/guidance/cg181/resources/surveillance-report-2018-cardiovascular-disease- 
risk-assessment-and-reduction-including-lipid-modification-2014-nice-guideline-cg181-pdf-

\section{$\underline{6123288665797}$}

Nordentoft, M., Wahlbeck, K., Hällgren, J., Westman, J., Ösby, U., Alinaghizadeh, H., ... \& Laursen, T. M. (2013). Excess mortality, causes of death and life expectancy in 270,770 patients with recent onset of mental disorders in Denmark, Finland and Sweden. PloS one, 8(1), e55176.

Olfson M, Gerhard T, Huang C, Crystal S, Stroup TS (2015). Premature mortality among adults with schizophrenia in the United States. JAMA Psychiatry 2015; 72:1172-81.

Patel, J. K., Buckley, P. F., Woolson, S., Hamer, R. M., McEvoy, J. P., Perkins, D. O., ... \& Cafe Investigators. (2009). Metabolic profiles of second-generation antipsychotics in early psychosis: findings from the CAFE study. Schizophrenia research, 111(1), 9-16.

Phutane, V. H., Tek, C., Chwastiak, L., Ratliff, J. C., Ozyuksel, B., Woods, S. W., \& Srihari, V. H. (2011). Cardiovascular risk in a first-episode psychosis sample: a 'critical period' for prevention?. Schizophrenia research, 127(1-3), 257-261.

Renwick, L., Drennan, J., Sheridan, A., Owens, L., Lyne, J., O'Donoghue, B., ... \& Clarke, M. (2017). Subjective and objective quality of life at first presentation with psychosis. Early intervention in psychiatry, $11(5), 401-410$.

Renwick, L., Jackson, D., Foley, S., Owens, E., Ramperti, N., Behan, C., ... \& Eadbhard, O. (2012). Depression and quality of life in first-episode psychosis. Comprehensive psychiatry, 53(5), 451-455.

Suttajit, S., \& Pilakanta, S. (2015). Predictors of quality of life among individuals with schizophrenia. Neuropsychiatric disease and treatment, 11, 1371.

Tek, C, Kucukgoncu, S, Guloksuz, S, Woods, SW, Srihari, VH, Annamalai, A. Antipsychotic-induced weight gain in first-episode psychosis patients: a meta-analysis of differential effects of antipsychotic medications. Early Intervention Psychiatry 2016; 10: 193-202.

Theodore, K., Johnson, S., Chalmers-Brown, A., Doherty, R., Harrop, C., \& Ellett, L. (2012). Quality of life and illness beliefs in individuals with early psychosis. Social psychiatry and psychiatric epidemiology, 47(4), 545-551.

Thongsai, S., Gray, R., Bressington, D. (2016). The physical health of people with schizophrenia in Asia: baseline findings from a physical health check programme. Journal of Psychiatric and Mental Health Nursing, 2016, 23, 255-266. DOI: 10.1111/jpm.12300

Tiihonen, J., Lönnqvist, J., Wahlbeck, K., Klaukka, T., Niskanen, L., Tanskanen, A., \& Haukka, J. (2009). 11year follow-up of mortality in patients with schizophrenia: a population-based cohort study (FIN11 study). The Lancet, 374(9690), 620-627. 
Vancampfort, D., Correll, C. U., Wampers, M., Sienaert, P., Mitchell, A., De Herdt, A., ... \& De Hert, M. (2014). Metabolic syndrome and metabolic abnormalities in patients with major depressive disorder: a meta-analysis of prevalences and moderating variables. Psychological medicine, 44(10), 2017-2028.

Vancampfort, D., Probst, M., Scheewe, T., Maurissen, K., Sweers, K., Knapen, J., \& De Hert, M. (2011). Lack of physical activity during leisure time contributes to an impaired health related quality of life in patients with schizophrenia. Schizophrenia research, 129(2-3), 122-127.

Vermeulen-Smit, E., Ten Have, M., Van Laar, M., \& De Graaf, R. (2015). Clustering of health risk behaviours and the relationship with mental disorders. Journal of affective disorders, 171, 111-119.

Von Elm, E., Altman, D. G., Egger, M., Pocock, S. J., Gøtzsche, P. C., \& Vandenbroucke, J. P. (2007). The Strengthening the Reporting of Observational Studies in Epidemiology (STROBE) statement: guidelines for reporting observational studies. Bulletin of the World Health Organization, 85, 867-872.

Weinmann S, Read J, Aderhold V. (2009). Influence of antipsychotics on mortality in schizophrenia: systematic review. Schizophrenia Research. 2009;113(1):1-11.

Whoqol Group. (1998). Development of the World Health Organization WHOQOL-BREF quality of life assessment. Psychological medicine, 28(3), 551-558.

World Health Organization. Division of Mental Health. (1996). WHOQOL-BREF: introduction, administration, scoring and generic version of the assessment: field trial version, December 1996. World Health Organization. https://apps.who.int/iris/handle/10665/63529.

World Health Organization Expert Consultation (2004). Appropriate body-mass index for Asian populations and its implications for policy and intervention strategies. Lancet. 2004;363(9403):157

Zhao, S., Xia, H., Mu, J., Wang, L., Zhu, L., Wang, A., \& Zhou, X. (2018). 10-year CVD risk in Han Chinese mainland patients with schizophrenia. Psychiatry research, 264, 322-326. 
Table 1 Socio-demographic and clinical characteristics

\begin{tabular}{|c|c|}
\hline Demographic & $\mathbf{N}(\%)$ \\
\hline \multicolumn{2}{|l|}{ Age } \\
\hline Mean (SD) & $40.42(10.82)$ \\
\hline Median (IQR) & $39(18)$ \\
\hline Range & $25-63$ \\
\hline \multicolumn{2}{|l|}{ Gender } \\
\hline Male & $41(50.6)$ \\
\hline Female & $40(49.4)$ \\
\hline \multicolumn{2}{|l|}{ Marital Status } \\
\hline Single & $38(46.9)$ \\
\hline Married & $29(35.8)$ \\
\hline Divorced, Widowed or others & $14(17.3)$ \\
\hline \multicolumn{2}{|l|}{ Educational Level } \\
\hline Primary or lower & $39(48.1)$ \\
\hline Secondary & $33(40.7)$ \\
\hline College, University or higher & $9(11.1)$ \\
\hline \multicolumn{2}{|l|}{ Employment Status } \\
\hline Unemployed & $26(32.1)$ \\
\hline Freelance or unemployed & $7(8.6)$ \\
\hline Housewife/Husband & $20(24.7)$ \\
\hline Employee & $12(14.8)$ \\
\hline Other & $16(19.8)$ \\
\hline \multicolumn{2}{|l|}{ Psychiatric Diagnosis } \\
\hline Other non-organic psychotic disorders (F28) & $38(46.9)$ \\
\hline Schizophrenia (F20) & $27(33.3)$ \\
\hline Acute and Transient psychotic disorder (F23) & $7(8.6)$ \\
\hline Schizoaffective disorders (F25) & $5(6.2)$ \\
\hline Unspecified non-organic psychosis (F29) & $4(4.9)$ \\
\hline \multicolumn{2}{|l|}{ Duration of illness (months) } \\
\hline Mean (SD) & $24.74(14.11)$ \\
\hline Median (IQR) & $24(24)$ \\
\hline Range & $12-60$ \\
\hline \multicolumn{2}{|l|}{ Prescribed Antipsychotics } \\
\hline \multicolumn{2}{|l|}{ Number of types of antipsychotics } \\
\hline Mean (SD) & $1.26(0.47)$ \\
\hline Median (IQR) & $1.0(1.0)$ \\
\hline Range & $1-3$ \\
\hline \multicolumn{2}{|l|}{ Antipsychotic Polypharmacy } \\
\hline Yes & $20(24.7)$ \\
\hline No & $61(75.3)$ \\
\hline
\end{tabular}


Second Generation Antipsychotics

Yes

$54(66.7)$

No

$27(33.3)$

Clozapine

Yes

8 (9.9)

No

$73(90.1)$

Long Acting Antipsychotic Injection

Yes

22 (27.2)

No

$59(72.8)$

Prescribed physical health treatments

Antihypertensives

Yes

$6(7.4)$

No

$75(92.6)$

Statins

Yes

$1(1.2)$

No

$80(98.8)$

Diabetes medications

Yes

$1(1.2)$

No

$80(98.8)$ 
Table 2: Cardiometabolic health risks, healthy lifestyle and Health related quality of life

\begin{tabular}{|c|c|c|}
\hline Variable & Mean (SD) & $\mathbf{N}(\%)$ \\
\hline \multirow[t]{2}{*}{ BMI } & $24.6(4.3)$ & \\
\hline & Range (17.0 to 35.1 ) & \\
\hline Underweight (<=18.4) & & $5(6.2)$ \\
\hline Normal (18.5-22.9) & & $28(34.6)$ \\
\hline Overweight (23-24.9) & & $16(19.8)$ \\
\hline Obese 1 (25-29.9) & & $22(27.2)$ \\
\hline Obese II $(>=30)$ & & $10(12.3)$ \\
\hline Pulse rate & $84.9(14.4)$ & \\
\hline Waist circumference measurement $(\mathrm{cm})$ & $85.5(9.6)$ & \\
\hline \multicolumn{3}{|l|}{ Waist circumference indicating central obesity } \\
\hline Males (\% of all males) & & $18(43.9)$ \\
\hline Females (\% of all females) & & $24(60.0)$ \\
\hline Blood pressure Systolic & $122.5(13.7)$ & \\
\hline Blood pressure Diastolic & $78.1(9.0)$ & \\
\hline QRISK Score & $2.4(2.7)$ & \\
\hline CVD individual Relative Risk & $1.9(1.6)[95 \% \mathrm{Cl}=1.6,2.3]$ & \\
\hline \multicolumn{3}{|l|}{ Range $=0.7-12.3$} \\
\hline \multicolumn{3}{|l|}{ Median=1.4 (IQR 1.35) } \\
\hline \multicolumn{3}{|l|}{ Healthy lifestyle behaviour risk areas } \\
\hline Smoking & & $23(28.4)$ \\
\hline Excessive Alcohol & & $8(9.9)$ \\
\hline Diet (insufficient fruit and vegetable consumption) & & $6(7.4)$ \\
\hline Diet (excessive fats consumption) & & $10(12.3)$ \\
\hline Insufficient Exercise & & $27(33.3)$ \\
\hline \multicolumn{3}{|l|}{ WHOQOL-BREF } \\
\hline Domain 1-Physical & $54.7(11.3)$ & \\
\hline Domain 2-Psychological & $56.9(11.8)$ & \\
\hline Domain 3-Social & $49.7(16.3)$ & \\
\hline Domain 4-Environmental & $61.6(11.7)$ & \\
\hline Overall quality of life & $3.3(0.9)$ & \\
\hline General health & $3.2(1.4)$ & \\
\hline
\end{tabular}

